Avrupa Bilim ve Teknoloji Dergisi

Özel Say1 26, S. 472-476, Temmuz 2021

(C) Telif hakkı EJOSAT'a aittir

Arasttrma Makalesi
European Journal of Science and Technology

Special Issue 26, pp. 472-476, July 2021

Copyright (C) 2021 EJOSAT

Research Article

\title{
Gri Kurt Optimizasyon Algoritması ile Kosinüs Modüleli Süzgeç Bankası Tasarımı
}

\author{
Sümeyya ARIKAN*, Fatma LATİFOĞLU² \\ ${ }^{1}$ Düzce Üniversitesi, Mühendislik Fakültesi, Biyomedikal Mühendisliği Bölümü, Düzce, Türkiye (ORCID: 0000-0001-7582-7537) \\ ${ }^{2}$ Erciyes Üniversitesi, Mühendislik Fakültesi, Biyomedikal Mühendisliği Bölümü, Kayseri, Türkiye (ORCID: 0000-0003-2018-9616)
}

(International Congress on Human-Computer Interaction, Optimization and Robotic Applications (HORA) 2021 - 11-13 June 2021)

(DOI: 10.31590/ejosat.960637)

ATIF/REFERENCE: Arıkan, S. \& Latifoğlu, F. (2021). Gri Kurt Optimizasyon Algoritması ile Kosinüs Modüleli Süzgeç Bankası Tasarımı. Avrupa Bilim ve Teknoloji Dergisi, (26), 472-476.

Öz

Bu çalışmada alt bant kodlama, görüntü, video veya ses sıkıştırma, spektral tahmin, biyolojik sinyal işleme ve uyarlanabilir sinyal işleme gibi pek çok alanda kullanılan çok kanallı tek düze neredeyse mükemmel kosinüs modüleli süzgeç bankası (NPR CMFB) tasarlanmıştır. Bu amaç kapsamında prototip süzgeç sonlu darbe yanıtlı süzgeç (FIR) yapısında tasarlanmıştır ve süzgeç katsayıları hedeflenen süzgeç bankasının tüm kanallarının frekans özellikleri kullanılarak gri kurt optimizasyon algoritması ve bu çalışma ile önerilen objektif fonksiyon kullanarak optimize edilmiştir. Süzgeç bankasının tasarlanması aşamasında amaç fonksiyon Tepe yeniden oluşturma hatası (PRE) ve hedeflenen süzgeç bankası ile tasarlanan süzgeç bankası arasındaki mutlak hatanın toplamı göz önünde bulundurularak elde edilmiştir. Tasarlanan filtrenin performansı durdurma bandı kazancı ve PRE parametreleri ile ortaya konmuştur.

Anahtar Kelimeler: Biyomedikal Sinyal İşleme, Kosinüs Modüleli Süzgeç Bankası, Gri Kurt Optimizasyon Algoritması.

\section{Cosine Modulated Filter Bank Design with Gray Optimization Algorithm}

\begin{abstract}
In this study, a multi-channel uniform almost perfect cosine modulated filter bank (NPR CMFB), which is used in many areas such as subband coding, image, video or audio compression, spectral prediction, biological signal processing, and adaptive signal processing, is designed. For this purpose, the prototype filter was designed in a finite pulse response filter (FIR) structure and the filter coefficients were optimized by using the frequency properties of all channels of the targeted filter bank using the gray wolf optimization algorithm and the objective function proposed in this study. In the design phase of the filter bank, the objective function has been obtained by considering the sum of the peak reconstruction error (PRE) and the absolute error between the targeted filter bank and the designed filter bank. The performance of the designed filter is demonstrated by the stopband gain and PRE parameters.
\end{abstract}

Keywords: Biomedical Signal Analysis, Cosine Modulation Filter Bank, Grey Wolf Optimization Algorithm

\footnotetext{
* Sümeyya ARIKAN: Düzce Üniversitesi, Mühendislik Fakültesi, Biyomedikal Mühendisliği Bölümü, Düzce, Türkiye (ORCID: 00000001-7582-7537), sumeyyaarikan@duzce.edu.tr
} 


\section{Giriş}

Biyomedikal sinyaller, vücuttan elde edilen hücre, organ ve sistem düzeyinde bilgiler taşıyan sinyallerdir. Mevcut sinyal işleme teknolojisi birden çok alt bileşeni içeren kanallara sahip olan bu sinyallerde çoklu kanalların elde edilmesine izin verir. Alt kanalların fizyolojik anlamlı etkileşimlerini analiz etmek ek sinyal işleme zorluklarını beraberinde getirmektedir [1]. Bu zorlukları çözebilmek amacıyla çok kanallı sinyal işleme teknikleri geliştirilmiştir. En çok kullanılan çok kanallı sinyal işleme tekniği süzgeç bankalarıdır [2].

Bir süzgeç bankası, sinyali daha etkili filtrelemek için farklı örneklem oranında alt bantlara ayırarak analiz eder (analiz bankası) ve bu alt bantları tekrar sentezleyerek (sentez bankası) orjinal sinyali oluşturur [2-5]. Sinyallerin farklı frekans bandlarına ayrılarak her bir kanalda farklı işlem yapılmasına müsaade eden süzgeç bankaları birçok alanda uygulanmıştır [56]. Çok kanallı süzgeç bankaları, frekans bantlarını eşit örnekleme oranıla düzenli ayırarak (UFB) veya farklı örnekleme oranlarıyla oluşturulan (NUFB) şeklinde tasarlanabilirler. Eşit olmayan frekans bantlarına ayrıştıran NUFB esneklik sebebiyle birçok uygulamada tercih edilir fakat yüksek dereceli süzgeçlerde tasarımı zordur [7]. Çok kanallı süzgeç bankaları sinyalin yeniden oluşturma kabiliyetine göre ikiye ayrılır. Bunlardan ilki mükemmel yeniden oluşturma (PR) süzgeç bankasıdır. PR süzgeç banklarında çıkış sinyali giriş sinyalinin bir kopyasıdır. PR biyomedikal sinyallerin sıkıştırılması ve yeniden genişletilmesi, görüntü işleme gibi birçok alanda önemli yer tutmaktadır. Bunun yanında ihtiyaç duyduğu süzgeçler sebebiyle hesaplama karmaşıklığı yüksektir ve bu süzgeçlerin hesaplaması verimli değildir. Hesap karmaşıklığının üstesinden gelmek amacıyla birçok uygulamada kullanılan neredeyse mükemmel (NPR) süzgeç bankaları giriş sinyalleri ile çıkış sinyalleri arasında hatanın olduğu tasarımlardır [8]. Neredeyse mükemmel süzgeç bankaları üç tip bozunmaya maruz kalır; örtüşme bozunumu (aliasing distortion), faz bozunumu (phase distortion), genlik bozunumu (amplitude distortion). Faz bozunumu, prototip süzgeçnin lineer faz özelliğine sahip süzgeçlerden seçilmesi ile minimize edilebilir. Örtüşme bozunumu fonksiyonu sıfıra yakınsatarak minimize edilebilir. Sıfır olması durumu PR tasarımlarda bulunur [9].

Literatürde süzgeç bankalarının biyomedikal sinyallerin analizinde kullanımına sıklıkla karşılaşılmaktadır. Sharma ve arkadaşları kalp krizi ile ilişkili EKG sinyalindeki kısa süre ve düşük büyüklükte değişiklikleri tespit edecek EKG tabanlı bir sistem kurmayı önermişlerdir. Tasarladıkları yeni iki bantlı optimal biorthogonal süzgeç bankası ile EKG sinyalleri altı alt banda ayrıştırmışlardır ve bu altı banttan istenilen tüm özellikleri elde etmişlerdir [10]. EKG sinyalinin analizi için Kumar ve arkadaşları uniform olmayan kosinüs modülasyonlu süzgeç bankalarının (CMFB) tasarımı için basit bir yineleme tekniği sunulmuştur. Önerilen teknik, optimizasyon için tek bir parametre kullanmıştır. Prototip süzgeci, Kaiser, Cosh ve Exponential gibi farklı ayarlanabilir pencere fonksiyonları ve sonlu dürtü yanıtı (FIR) dijital süzgeç tasarım tekniği kullanılarak tasarlanmıştır. Bu yöntemde, süzgeç katsayılarını ayarlamak için kesme frekansı veya geçiş bantı kenar frekansı değiştirileceği, böylece yeniden yapılandırma hatası en aza indirilebileceği ortaya konmuştur [11]. Kalathil ve Elias dijital işitme cihazı uygulamaları için tek tip olmayan CMFB ile verimli ve basit tasarımları önerilmiştir. Çeşitli odyogram türleri için alt bantlar ve eşleştirme hatasının iyileştirilmiş performans aralıkları karşılaştırılmış, daha az uygulama karmaşıklığı ve ayarlama işleminde daha fazla esneklik sağlanmıştır [12]. EEG temelli önlemlerle depresyon tespit sisteminin geliştirilmesini amaçlandığı bir sistemde yeni tasarlanan bant genişliği süresi lokalize üç kanallı ortogonal dalgacık süzgeç bankası (OWFB) kullanılmıştır. EEG sinyali, en uygun altı uzunlukta bir OWFB kullanılarak altı dalgacık alt-bantlarına (WSB) ayrıştırılarak analiz edilmiş, depresyon sınıflandırmada ortalama sınıflandırma yüzdesine ulaşılmıştır [13]. Basit prototip süzgeç tasarımı ve ayrık kosinüs sinyali kullanılması avantajı sebebiyle birçok uygulama kosinüs modüleli süzgeç bankalarına odaklanmıştır [6-10]. Prototip süzgeç tasarımı CMFB nin en önemli basamağıdır. Dolayısıyla prototip süzgeç seçimi ve katsayılarının hesabı önemlidir. Gerçekleştirilen çalışmada prototip filtre CMFB na ait tüm kanalların frekans özellikleri kullanılarak yeni bir yaklaşımla optimize edilmiştir. Bu amaç kapsamında Kaiser penceresine ait beta parametresinin optimizasyonu ile prototip FIR filtre elde edilmiştir.

\section{Materyal ve Metot}

Kosinüs modüleli süzgeç bankası tek bir prototip süzgecin modülasyonu ile elde edilen tasarımının kolay ve verimli olması sebebiyle son yıllarda çokça tercih edilmektedir. Bu çalışmada düzenli neredeyse mükemmel yeniden oluşturma kosinüs modüleli süzgeç bankası tasarımı amaçlanmıştır. Tek-düze NPR CMFB nin prototip süzgeci FIR yapıda oluşturulmuş, ayrık kosinüs dönüşümü ile modülasyonu sağlanmıştır. Süzgeç katsayılarının optimizasyonu literatürdeki prototip süzgeç katsayılarının optimizasyonu kullanımlarından farklı olarak tüm süzgeç bankası frekans kanalları kullanılarak elde edilmiştir. FIR filtrenin beta parametresinin optimizasyonu için ise gri kurt optimizasyon algoritması kullanılmıştır.

\subsubsection{Kosinüs Modlüleli Süzgeç Bankası}

M-kanallı süzgeç bankalarının özel bir alt sınıfı olan kosinüs modüleli süzgeç bankaları, giriş işaretinin $\mathrm{x}(\mathrm{n})$ frekans spektrumunu $\mathrm{M}$ adet alt banda ayırır. $\mathrm{M}$ oranında örnek seyreltme yapılarak (analiz kısmı) elde edilen alt bantlar sayesinde daha verimli sinyal işleme, depolama ve veri iletimi için alt işlemlerin yapılmasını sağlanmaktadır. Alt bant sinyali $\mathrm{M}$ oranında örnek artırma yapılarak sentez süzgecinden geçirilir ve giriş sinyali yeniden, y(n), oluşturulmaktadır (Şekil 1).

CMFB'lerde bir prototip süzgecin katsayılarının modülasyonu yapılarak $H_{k}(z)$ analiz süzgeçleri ve $F_{k}(z)$ sentez süzgeçleri elde edilir (Denklem 1-2) 


$$
\begin{aligned}
& h_{k}(n)=2 h(n) \cos \left[(2 k+1) \frac{\pi}{2 M}\left(n-\frac{N}{2}\right)+(-1)^{k} \frac{\pi}{4}\right] \\
& f_{k}(n)=2 h(n) \cos \left[(2 k+1) \frac{\pi}{2 M}\left(n-\frac{N}{2}\right)-(-1)^{k} \frac{\pi}{4}\right] \\
& 0 \leq n \leq N-1, \quad 0 \leq k \leq M-1
\end{aligned}
$$

Verilen eşitliklerde $N$ süzgeç derecesini $h(n)$ prototip süzgecin dürtü yanıtı katsayılarını vermektedir. Giriş sinyali ve çıkış sinyali arasındaki ilişki şu şekildedir [9]:

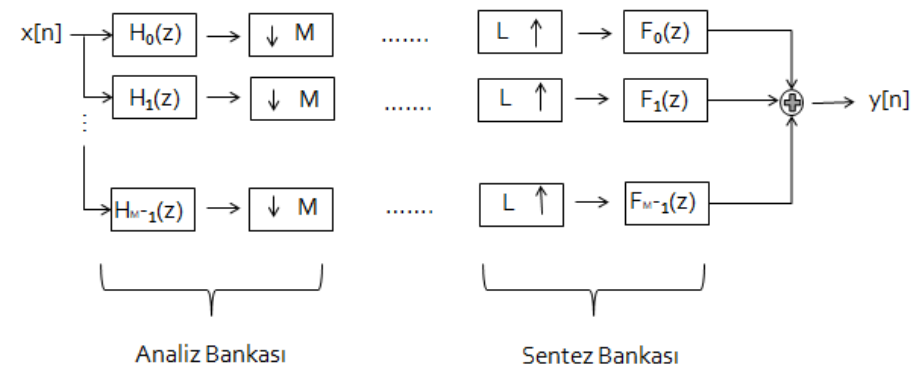

Şekil 1. M kanallı süzgeç bankası [4]

$$
\begin{aligned}
& Y(z)=T_{0}(z) X(z)+\sum_{l=1}^{M-1} T_{1}(z) X\left(z e^{-\frac{j 2 \pi 1}{M}}\right) \\
& T_{0}(z)=\frac{1}{M} \sum_{k=0}^{M-1} F_{k}(z) H_{k}(z) \\
& T_{1}(z)=\frac{1}{M} \sum_{k=0}^{M-1} F_{k}(z) H_{k}\left(z e^{-j 2 \pi l / M}\right) \quad l=1,2 . . M-1
\end{aligned}
$$

Yukarıda verilen eşitliklerde,

$$
\left.\left.H_{\mathrm{k}}(z)=\sum_{n=0}^{N-1} h_{k}(n) z^{-n}\right), \quad F_{\mathrm{k}}(z)=\sum_{n=0}^{N-1} f_{k}(n) z^{-n}\right)
$$

olup $\mathrm{T}_{0}(\mathrm{z})$ bozulma transfer fonksiyonu (eşitlik 4) ve $\mathrm{T}_{1}(\mathrm{z})$ örtüşme transfer fonksiyonudur (eşitlik 5) [9]. CMFB de aşağıdaki eşitlik kullanılırsa mükemmel yeniden oluşturma mümkündür [10]:

$$
\left|H_{0}\left(e^{j \omega}\right)\right|^{2}+\left|H_{0}\left(e^{j(\omega-\pi / M}\right)\right|^{2}=1, \quad 0<\omega \leq \pi / M
$$

Bu eşitlik eğer $\omega=\pi / 2 M$ olarak değerlendirilirse;

$$
\begin{aligned}
& \left|H_{0}\left(e^{j \pi / 2 M}\right)\right|^{2}+\left|H_{0}\left(e^{j(\pi / 2 M-\pi / M}\right)\right|^{2}=1, \\
& \left|H_{0}\left(e^{j \pi / 2 M}\right)\right|=0.707
\end{aligned}
$$

Bir NPR prototip süzgeç tasarımı yukarıdaki şartlar yaklaşık olarak gerçekleştirildiğinde sağlanır. Küçük genlik ve örtüşme bozulmalarına izin veren NPR tasarımı, PR süzgeç bankasına oranla önemli ölçüde geliştirilmiş durdurma bandı performansı sağlamıştır [14].

Prototip süzgeç tasarımında sonlu dürtü yanıtlı ve sonsuz dürtü yanıtlı (IIR) süzgeçler kullanılmaktadır. IIR süzgeçler düşük derecelerde gerçekleştirilebilmesi sebebiyle avantajlı olmasına rağmen kararlılık ve doğrusal olmayan faz yanıtına sahip olması gibi dez avantanjlarına sahiptir. Ancak FIR süzgeçler daima kararlı olma ve doğrusal faz yanıtına sahip olma avantajları sebebiyle yaygın olarak kullanılmaktadır.

FIR süzgece ait dürtü yanıtı $(\mathrm{h}[\mathrm{n}])$ aşağıda verilen eşitlikte tanımlanmaktadır.

$$
h[n]=\sum_{k=0}^{\mathrm{N}-1} b_{k} \delta[n-k]
$$

Bu eşitlikte $b_{k}$ FIR süzgeç katsayılarını göstermektedir ve N ise süzgecin derecesini göstermektedir. FIR süzgeç tasarımı amaçlı literatürde çeşitli yöntemler bulunmakta olup bu yöntemler arasında en yaygın olan yöntem pencere fonksiyonu kullanarak pencerelenmiş FIR süzgeç tasarımıdır [15] . Pencerelenmiş FIR süzgeç tasarımına ait dürtü yanıtı Denklem 10 da verilmektedir.

$$
h_{w}[n]=h_{d}[n] w_{k}[n]
$$

Pencerelenmiş FIR süzgeç tasarımında ise parametreleri ayarlanabilen FIR süzgeç tasarımı esnek yapıya sahip olması nedeniyle tercih edilmektedir. Gerçekleştirilen çalışmada Kaiser penceresi kullanarak prototip filtre tasarlanmıştır. Kaiser penceresi Denklem 11 da ifade edildiği gibi tanımlanmaktadır.

$w_{k}[n]=\frac{I_{0}\left(b_{k} \sqrt{1-\left(\frac{2 n}{N-1}\right)^{2}}\right)}{I_{0}\left(b_{k}\right)}|n| \leq(N-1) / 2 i c ̧ i n$

Verilen eşitlikte $\mathrm{N}$ pencere uzunluğunu, $b_{k}$ ayarlanabilir bağımsız parametreyi (beta parametresi) ve $I_{0}(x)$ sıfır dereceli birinci tür geliştirilmiş Bessel fonksiyonunu göstermektedir.

$\mathrm{Bu}$ çalışmada prototip FIR filtre Kaiser penceresine ait beta parametresi ayarlanarak, Şekil 2 de görüldüğü gibi tasarlanması planlanan CMFB ve tasarlanan süzgeç bankası arasındaki mutlak hatanın toplamı ve PRE minimize edilmeye çalışılarak tasarlanmıştır.

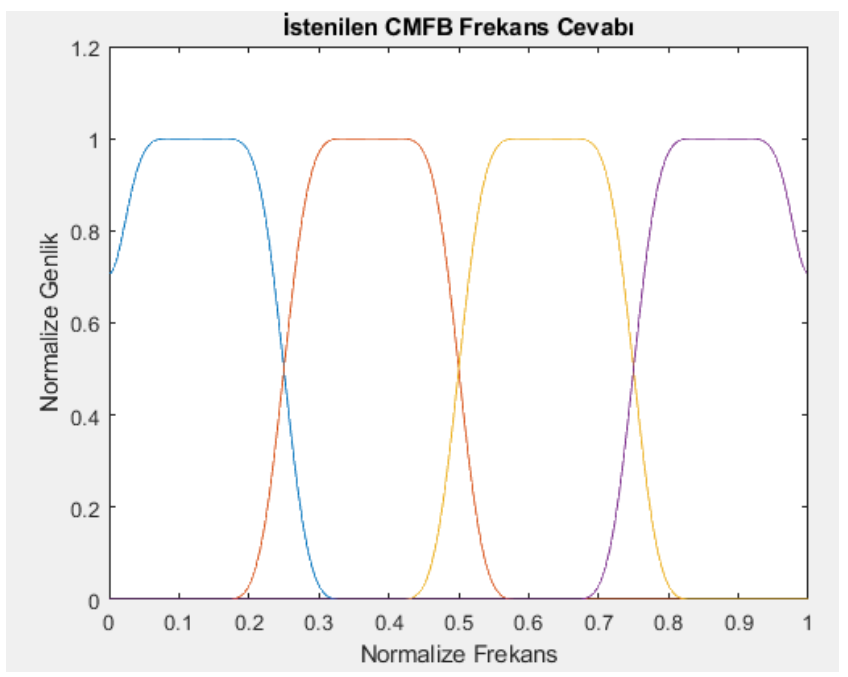

Şekil 2. Tasarlanması planlanan süzgeç bankası frekans cevabı

CMFB tasarımında kullanılan amaç fonksiyonuna ait eşitlik aşağıda yer almaktadır.

$$
e_{f}=P R E+\sum_{i=1}^{M}\left|H d_{i}\left(e^{j w}\right)-H o_{i}\left(e^{j w}\right)\right|
$$

Eşitlik 12 de verilen $H d_{i}\left(e^{j w}\right)$ i inci kanala ait istenen frekans yanıtı ve $H o_{i}\left(e^{j w}\right)$ i inci kanala ait elde edilen frekans yanıtıdır. 


\subsubsection{Gri Kurt Optimizasyon Algoritması}

Gri kurt optimizasyon algoritmas1, besin zincirinin en üstünde yer alan gri kurtların (canis lupus) liderlik ve avlanma hiyerarşisinden yola çıkarak oluşturulmuş bir sürü tabanlı metasezgisel optimizasyon algoritmasıdır. 5-10 bireylik sürülerden oluşan gri kurtların liderlik hiyerarşisini taklit etmek için dört gri kurt grubu bulunmaktadır. Bunlar hiyerarşinin tepesinde yer alandan aşağıya doğru; $\operatorname{alfa}(\alpha)$, beta $(\beta)$, delta $(\delta)$ ve omega $(\omega)$ olarak adlandırılırlar. Kurtların avlanma alışkanlıkları benzetiminde ise av arama, avı kuşatma ve ava saldırma adımları uygulanmaktadır [16]. Gri kurtlar avlarına ait yerleri belirler ve alfa kurt öncülügüunde kuşatır. GWO da omega kurdu bulunmamaktadır ve soyut arama alanında avın yeri hakkında bilgi olmadığı için azalan sırada alfa (en iyi çözüm), beta ve delta kurtlarının avın potansiyel yeri hakkında bilgiye sahip olduğu varsayılıp en iyi üç çözüm olarak kaydedilir. Kurtların konumu bu ilk üç çözüme göre belirlenir [17].

GWO algoritmasının kaba kodu şu şekildedir [18]:

- Başlangıç populasyonunu ayarla

- Uygunluk fonksiyonlarını hesapla, ilk üç çözümü $\alpha, \beta$, $\delta$ olarak kaydet

- $\quad$ while (iterasyon sayısı $<$ maximum iterasyon sayısı)

- $\grave{a}$ yı azalt

- for (her gri kurt için)

- $\alpha, \beta, \delta$ vektörlerini oluştur

- Mesafe vektörlerini hesapla

- Deneme vektörlerini hesapla

- Gri kurdun durumunu güncelle

- $\quad$ En kötü kurdu ele, yerine yeni bir kurt üret

- Seçilen kurtları güncelle, yinelemeyi bir artır

- end while

- Alfa kurdu döndür

\subsubsection{Tasarım Parametreleri}

Kosinüs modüleli süzgeç bankası tasarımı MATLAB ortamında Intel Core 2.2 GHz işlemcili 8 GB RAM e sahip bilgisayarda yapılmıştır. Süzgeç bankasına ait prototip süzgeç FIR süzgeç, GWO algoritması ile tasarlanmıştır. Süzgeç bankası 4 kanallı tekdüze neredeyse mükemmel yeniden oluşturma yöntemine göre hazırlanmıştır. Önerilen CMFB farklı süzgeç derecelerinde gerçekleştirilmiştir. Kaiser penceresine ait beta parametresi GWO algoritması ile optimize edilmiştir. Ayrıca literatürde yaygın olarak kullanılan Parks McCllein algoritması kullanarak elde edilen CMFM nın performans sonuçları ile GWO tabanlı CMFB performansları kıyaslanmıştır. Süzgeç bankasının performans değerlendirilmesinde $A_{s}$ durduma bandı kazancı ve PRE yeniden oluşturma hatası kullanılmıştır:

$$
A_{s=}-20 * \log _{10}\left|H_{0}(\omega)\right| \quad \omega=\omega_{s}
$$

olup, $\omega_{s}$ burada durdurma bandı frekansıdır. Gerşekleştirilen çalışmada $\omega_{s}$ durdurma bandı frekansı prototip filtrenin normalize frekans yanıtının 0.001 değerine düştüğü frekans değeri olarak tanımlanmıştır. PRE için ise aşağıdaki eşitlik kullanılmıştır:

$$
P R E=\max \left\{10 * \log \left(\sum_{i=1}^{M}\left|H_{0}\left(e^{j \omega}\right)\right|^{2}\right)\right.
$$

Burada M kanal sayısını ve $H_{0}\left(e^{j \omega}\right)$ CMFB na ait kanalların frekans yanıtını göstermektedir.

\section{Araştırma Sonuçları ve Tartışma}

\subsection{Sonuçlar ve Tartışma}

$\mathrm{Bu}$ çalışmada GWO algoritmasıyla tasarlanan 4 kanallı tekdüze neredeyse mükemmel CMFB; 20, 32, 64 ve 128 süzgeç derecelerine göre değerlendirilmiştir. Önerilen tasarım Parks McCllein algoritmasıyla tasarlanan CMFB ye ait performans parametreleriyle kıyaslanmıştır. Tablo 1 de verilen her bir $\mathrm{N}$ derecesi için elde edilen $A_{s}$ ve PRE değerlerine bakıldığında GWO tabanlı CMFB nın performansının literatürde yaygın olarak kullanılan Parks McCllein algoritmasıyla tasarlanan CMFB na göre oldukça yüksek performanslı olduğu ortaya konulmuştur. Süzgeç derecesi 64'ü geçtiğinde durdurma bandı kazancı önemli derecede arttığı görülmektedir.

Aşağıda N=64 derecesiyle tasarlanan süzgeç bankasına ait prototip FIR süzgeç grafiği (Şekil 3.a) ve tasarlanan dört kanallı CMFB süzgeç grafiği (Şekil 3.b) gösterilmiştir. Elde edilen sonuçlara göre beta parametresi optimize edilmiş Kaiser penceresi ile tasarlanan CMFB na ait performans parametreleri As ve PRE değerleri sırasıyla 20 dereceden filtre için 41.8980 ve 5.6696e-05, 32. Dereceden filtre için 49.5920 ve 3.2624e-05, 64. Dereceden filtre için 83.1566 ve $5.4547 \mathrm{e}-08,128$. Dereceden filtre için 111.3054 ve $7.8623 e-05,256$. Dereceden filtre için 109.4588 ve 4.3889 e-05 olarak elde edilmiştir. Elde edilen sonuçlar incelendiğinde GWO tabanlı CMFB nın yüksek performanslı olduğu görülmektedir.

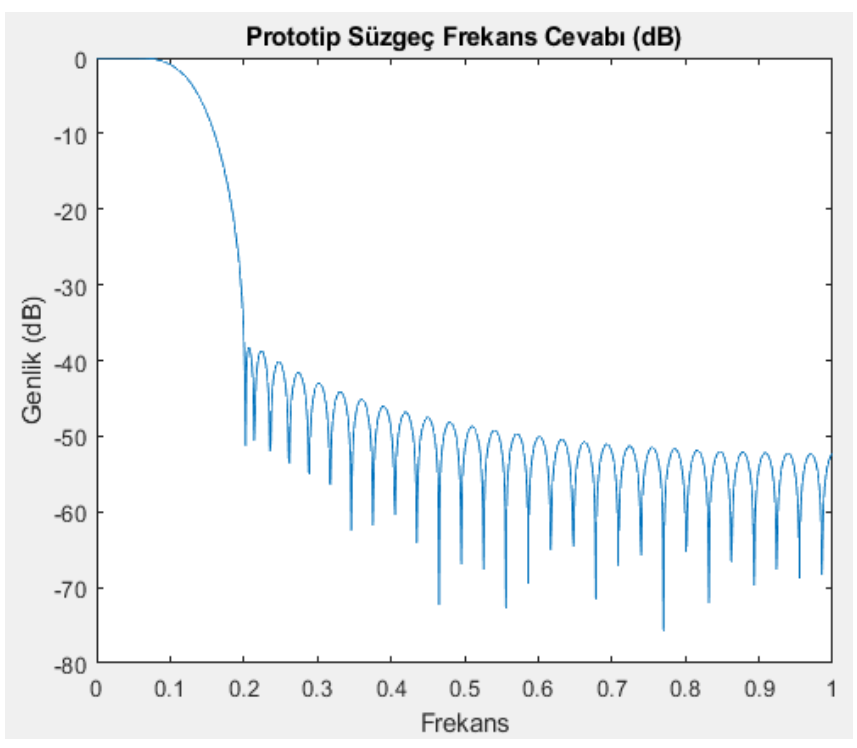

Şekil 3. 64. Dereceden prototip süzgeç frekans cevab1 


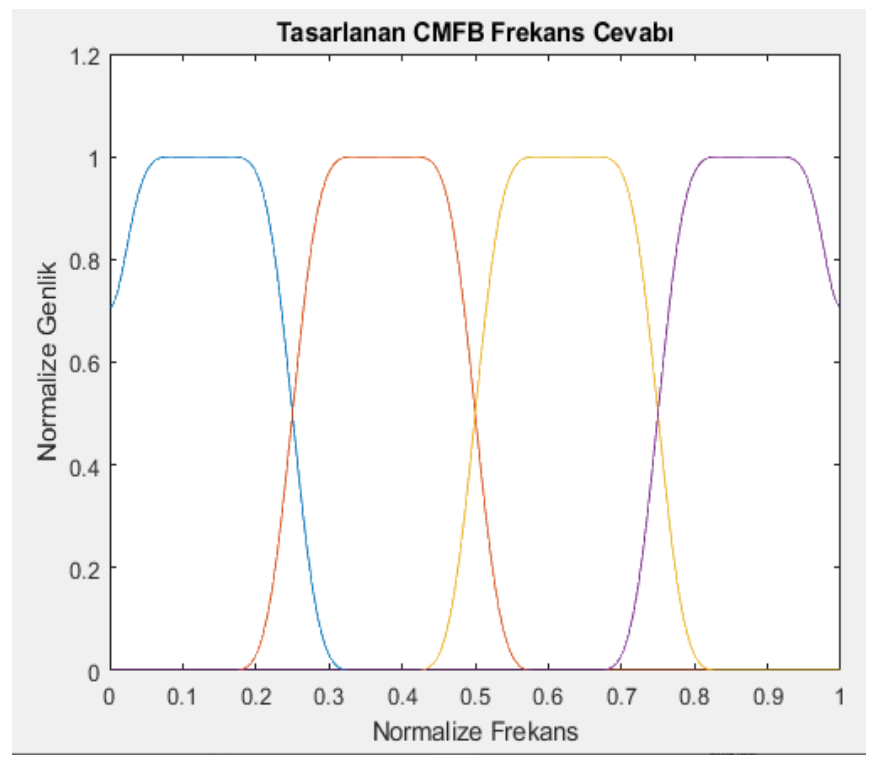

Şekil 4. Tasarlanan CMFB süzgeç frekans cevabı

Tablo 1. Parks McClein ve Önerilen algoritma kullanılarak elde edilen 4 Kanallı CMFB performans parametreleri

\begin{tabular}{|c|c|c|c|c|}
\hline & \multicolumn{2}{|c|}{ Parks McCllein } & \multicolumn{2}{c|}{ GWO (Önerilen metod) } \\
\hline $\mathbf{N}$ & $\boldsymbol{A}_{\boldsymbol{s}}(\boldsymbol{d B})$ & PRE & $\boldsymbol{A}_{\boldsymbol{s}}(\mathbf{d B})$ & PRE \\
\hline 20 & 24.3229 & 0.0314 & 41.8980 & $5.6696 \mathrm{e}-05$ \\
\hline 32 & 25.0655 & 1.5420 & 49.5920 & $3.2624 \mathrm{e}-05$ \\
\hline 64 & 24.7415 & 1.0307 & 83.1566 & $5.4547 \mathrm{e}-08$ \\
\hline 128 & 24.8004 & 0.8022 & 111.3054 & $7.8623 \mathrm{e}-05$ \\
\hline 256 & 31.9243 & 0.6886 & 109.4588 & $4.3889 \mathrm{e}-05$ \\
\hline
\end{tabular}

\section{Teşekkür}

Bu çalışma Erciyes Üniversitesi Bilimsel Araştırma Projeleri Koordinasyon birimince desteklenmiştir (Proje numarası: FDK2019-8760)

\section{Kaynakça}

1. Kutz, M. (2003). Standard handbook of biomedical engineering and design (p. 2). New York: McGraw-Hill.

2. Milic, L. (2009). Multirate filtering for digital signal processing: MATLAB aplications (First Edition). Information Sicence Reference

3. Mitra, S., M. (2006) Digital signal processing: a computerbased approach (Third Edition). McGraw Hill Higher Education.

4. Vaidyanathan, P. P. (1993). Multirate systems and filter banks. Pearson Education India.

5. Dolecek, G. J. (Ed.). (2017). Advances in Multirate Systems. Springer.
6. Chandra, S., Sharma, A., \& Singh, G. K. (2019). Computationally Efficient Cosine Modulated Filter Bank Design for ECG Signal Compression. IRBM.

7. Sharma, I., Kumar, A., \& Singh, G. K. (2016). Adjustable window based design of multiplier-less cosine modulated filter bank using swarm optimization algorithms. AEUInternational Journal of Electronics and Communications, 70(1), 85-94.

8. Kumar, A., \& Kuldeep, B. (2012). Design of M-channel cosine modulated filter bank using modified Exponential window. Journal of the Franklin Institute, 349(3), 13041315.

9. G. Özdemir And N. Karaboğa, "Uniform cosine modulated filter banks - a new cascade method based on window functions," Journal of The Faculty Of Engineering And Architecture Of Gazi University, vol.35, pp.403-418, 2019

10. Sharma, M., San Tan, R., Acharya, U. R. A novel automated diagnostic system for classification of myocardial infarction ECG signals using an optimal biorthogonal filter bank. Computers in biology and medicine. 2018.

11. Kumar, A., Singh, G. K., Anurag, S. An optimized cosinemodulated nonuniform filter bank design for subband coding of ECG signal. Journal of King Saud UniversityEngineering Sciences, 27(2), 158-169, 2015.

12. Kalathil, S., \& Elias, E. (2015). Efficient design of nonuniform cosine modulated filter banks for digital hearing aids. AEU-International Journal of Electronics and Communications, 69(9), 1314-1320

13. Sharma, M., Achuth, P. V., Deb, D., Puthankattil, S. D., Acharya, U. R. An automated diagnosis of depression using three-channel bandwidth-duration localized wavelet filter bank with EEG signals. Cognitive Systems Research, 52, 508-520. 2018

14. Viholainen, A., Saramaki, T., Renfors, M. (1999, September). Nearly perfect-reconstruction cosinemodulated filter bank design for VDSL modems. In ICECS'99. Proceedings of ICECS'99. 6th IEEE International Conference on Electronics, Circuits and Systems (Cat. No. 99EX357) (Vol. 1, pp. 373-376). IEEE.

15. Latifoğlu, F., (2020). A novel singular spectrum analysisbased multi-objective approach for optimal FIR filter design using artificial bee colony algorithm. Neural Computıng \& Applications , vol.32, no.17, 13323-13341.

16. Das, P., Das, A. (2020, December). Adaptive Gabor Filtering using Grey Wolf Optimization for Enhancement of Brain MRI. In 2020 IEEE International Women in Engineering (WIE) Conference on Electrical and Computer Engineering (WIECON-ECE) (pp. 356-359). IEEE.

17. Wang, J. S., Li, S. X. (2019). An improved grey wolf optimizer based on differential evolution and elimination mechanism. Scientific reports, 9(1), 1-21.

18. Mirjalili, S., Mirjalili, S. M., \& Lewis, A. (2014). Grey Wolf Optimizer. Advances in Engineering Software, 69, 4661. 